\title{
DETERMINAÇÃO TURBIDIMÉTRICA DE METILBROMETO DE HOMATROPINA EM FORMULAÇÕES FARMACÊUTICAS EMPREGANDO UM SISTEMA DE ANÁLISE POR INJEÇÃO EM FLUXO
}

Larissa S. Canaes e Orlando Fatibello-Filho*

Departamento de Química, Centro de Ciências Exatas e de Tecnologia, Universidade Federal de São Carlos, CP 676, 13560-970 São Carlos - SP, Brasil

Recebido em 17/10/05; aceito em 14/3/06; publicado na web em 25/7/06

\begin{abstract}
TURBIDIMETRIC DETERMINATION OF HOMATROPINE METHYLBROMIDE IN PHARMACEUTICAL FORMULATIONS USING A FLOW INJECTION ANALYSIS SYSTEM. A flow injection turbidimetric procedure exploiting merging zones is proposed for determining homatropine methylbromide (HMB) in pharmaceutical preparations. The determination is based on the precipitation reaction of homatropine methylbromide with $\mathrm{AgNO}_{3}$ solution to form a precipitate, which was measured at $410 \mathrm{~nm}$. The analytical curve was linear in the HMB concentration range from $8.0 \times 10^{-4}$ to $1.7 \times 10^{-3} \mathrm{~mol} \mathrm{~L}^{-1}$, with a detection limit of $9.5 \times 10^{-5} \mathrm{~mol} \mathrm{~L}^{-1}$. The recoveries ranged from 94.9 to $104 \%$, the sampling frequency was $75 \mathrm{~h}^{-1}$ and relative standard deviations were smaller than $2.0 \%$ for solutions containing $1.2 \times 10^{-3}$ and $1.5 \times 10^{-3} \mathrm{~mol} \mathrm{~L}^{-1} \mathrm{HMB}(\mathrm{n}=10)$. The results obtained for commercial formulations using the FIA procedure were in good agreement with those obtained by using a comparative method $(r=0.9983)$.
\end{abstract}

Keywords: homatropine methylbromide; flow injection; turbidimetry.

\section{INTRODUÇÃO}

Os compostos antimuscarínicos são fármacos que atuam no sistema nervoso parassimpático sendo atropina, escopolamina, eucatropina, homatropina e metilbrometo de homatropina $(\mathrm{MBH})$ as mais amplamente usadas.

A atropina foi isolada pela primeira vez por Mein em $1831 \mathrm{e}$ seus efeitos foram estudados principalmente durante a segunda metade do século XIX ${ }^{1}$. Por muitos séculos, os povos antigos utilizaram preparados de beladona (Atropa belladonna) para diversos fins terapêuticos ${ }^{2}$.

Os fármacos antimuscarínicos semi-sintéticos ou sintéticos são de estrutura química diversa, quase todos são ésteres com grupo aromático na molécula. A existência de um nitrogênio terciário ou quaternário condiciona diferenças farmacocinéticas na sua potência e também em sua ação ganglionar. Entre os fármacos com nitrogênio terciário, destacam-se diciclomina, homatropina, tropicamida etc. Entre aqueles contendo nitrogênio quaternário, destacam-se metilbrometo de homatropina, pinavério, butilescopolamina, isopropamida entre outros ${ }^{2}$. O metilbrometo de homatropina é originado da adição de um segundo radical metila à molécula de homatropina.

As drogas antimuscarínicas mais amplamente usadas são atropina, ciclopentolato, escopolamina, eucatropina, homatropina, metilbrometo de homatropina e tropicamida.

A Figura 1 apresenta as fórmulas estruturais da atropina, homatropina e metilbrometo de homatropina ${ }^{1,2}$.

$\mathrm{O} \mathrm{MBH}$ é utilizado, freqüentemente, em distúrbios gastrintestinais como antiespasmódico, agindo diretamente sobre o músculo liso. A dose para adultos, por via oral, é de 12 a $16 \mathrm{mg} / \mathrm{kg}$ de peso corpóreo dia e, em crianças, $0,1 \mathrm{mg} / \mathrm{kg}$ de peso corpóreo dia. Efeitos adversos em pacientes incluem rubor facial, constipação, retenção urinária, diminuição da sudorese e da secreção lacrimal, secura na boca, irritabilidade, dilatação pupilar, entre outros sintomas dependentes da ação anticolinérgica ${ }^{3}$.

*e-mail: bello@dq.ufscar.br

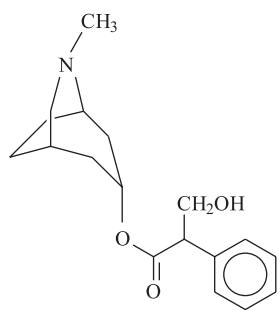

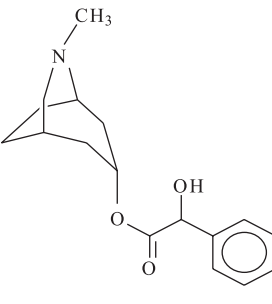

B

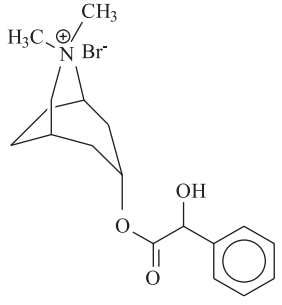

Figura 1. Fórmulas estruturais da atropina (A), homatropina (B) $e$ metilbrometo de homatropina $(C)$

Rengstorff e Doughty ${ }^{4}$ relataram as possíveis complicações neurológicas determinadas pelo uso oftalmológico de cicloplégicos e midriáticos (incluindo a homatropina). A sintomatologia inclui desde cefaléia, tremor, fraqueza e sonolência, até confusão, alucinação, disartria (dificuldade na articulação das palavras, resultante de perturbação nos centros nervosos), ataxia (falta de coordenação dos membros do corpo), crises epilépticas, coma e morte. Tune et $a l .{ }^{5}$ chamaram a atenção dos oftalmologistas para a possibilidade de ocorrerem manifestações de intoxicação do sistema nervoso central, determinando quadro de delírio prolongado, alucinações, disartria e mania, com o uso tópico ocular de homatropina, mesmo em concentrações de $5 \% \mathrm{~m} / \mathrm{v}$.

Poucos são os métodos descritos na literatura para determinação de metilbrometo de homatropina, a saber: cromatografia líquida de alto desempenho (HPLC) ${ }^{6}$, cromatografia gás-líqui$\mathrm{do}^{7}$ e espectrofotometria de absorção na região do ultra-violeta ${ }^{8}$ e visível ${ }^{9}$.

O procedimento da Farmacopéia Brasileira ${ }^{10}$ recomenda um método potenciométrico empregando-se como titulante nitrato de prata, eletrodo indicador de prata metálica e como referência um eletrodo de prata/cloreto de prata com dupla junção.

A Farmacopéia Americana (USP) ${ }^{11}$ recomenda titulação com ácido perclórico. 
Uma vez que esses fármacos têm sido empregados em diversas situações clínicas, tornou-se necessário o desenvolvimento de procedimentos analíticos simples, rápidos e eficazes para monitoramento. Desenvolveu-se neste trabalho um sistema de análises por injeção em fluxo, com zonas coalescentes, para a determinação turbidimétrica de metilbrometo de homatropina em formulações farmacêuticas, empregando solução de nitrato de prata como reagente.

\section{PARTE EXPERIMENTAL}

\section{Instrumentação}

As medidas de turbidez foram feitas utilizando espectrofotômetro (Femto, Brasil) modelo 432, equipado com uma cela de fluxo de $1,0 \mathrm{~cm}$ de caminho ótico, em $410 \mathrm{~nm}$, conectado a um registrador $\mathrm{x}$ t, de dois canais Cole Parmer (Niles - IL, USA), modelo 1202. As demais medidas espectrofotométricas foram feitas em espectrofotômetro com arranjo linear de diodos Hewlett Packard, modelo 8452A, empregando-se uma cubeta de quartzo de $1,0 \mathrm{~cm}$ de caminho óptico acoplado a um microcomputador. Para propulsão das soluções de referência, reagentes e amostras utilizou-se uma bomba peristáltica de 12 canais (Ismatec, Zurich - Suíça), modelo 7618-50 e tubos de propulsão de Tygon ${ }^{\circledR}$. Para confecção das bobinas reacionais, alça de amostragem, alça de reagente e do percurso analítico foram empregados tubos e conexões de polietileno com diâmetros internos de $0,8 \mathrm{~mm}$. As amostras, soluções de referência e reagentes foram inseridas no sistema de fluxo com auxílio de um injetor comutador manual, construído em acrílico ${ }^{12}$.

No método comparativo ${ }^{10}$ foi utilizado um eletrodo de prata metálica como eletrodo indicador e eletrodo de referência com dupla junção de $\mathrm{Ag} / \mathrm{AgCl}\left(\mathrm{KCl} 3,0 \mathrm{~mol} \mathrm{~L}^{-1}\right)$ e $\mathrm{NaNO}_{3} 3,0 \mathrm{~mol} \mathrm{~L}^{-1}$.

\section{Reagentes e soluções}

Todos os reagentes empregados neste trabalho foram de grau analítico e as soluções foram preparadas com água destilada e tratada em um sistema Milli-Q, modelo UV-Plus ultra-baixo teor de compostos orgânicos dissolvidos, Millipore (Redford, MA, USA). A solução estoque de metilbrometo de homatropina $500 \mathrm{mg} \mathrm{L}^{-1}$ foi preparada pela dissolução de $12,5 \mathrm{mg}$ de metilbrometo de homatropina (Henrifarma) em balão volumétrico de $25 \mathrm{~mL}$. A solução de $\mathrm{AgNO}_{3} 1,0 \times 10^{-2} \mathrm{~mol} \mathrm{~L}^{-1}$ (Sigma, USA) foi preparada pela dissolução de $340 \mathrm{mg}$ deste reagente em balão volumétrico de 200 $\mathrm{mL}$ com água desionizada.

\section{Preparação das amostras}

O metilbrometo de homatropina foi determinado em amostras de formulações farmacêuticas adquiridas em farmácias locais. As amostras foram preparadas pela dissolução de 2,0 e 2,5 mL das amostras A e B, respectivamente, em um balão de $10 \mathrm{~mL}$, sendo o volume completado com água desionizada.

\section{Sistema de análises por injeção em fluxo}

O diagrama esquemático do sistema em fluxo com zonas coalescentes é mostrado na Figura 2.

Alíquotas de $375 \mu \mathrm{L}$ (alça de $75 \mathrm{~cm}$ ) de soluções de referência ou da amostra e de $25 \mu \mathrm{L}$ (alça de $5 \mathrm{~cm}$ ) da solução do reagente foram injetadas simultaneamente no transportador $\left(\mathrm{H}_{2} \mathrm{O}\right)$ utilizando-se um injetor comutador manual. Soluções de metilbrometo de homatropina de $1,5 \times 10^{-3}, 2,0 \times 10^{-3}$ e $2,5 \times 10^{-3} \mathrm{~mol} \mathrm{~L}^{-1}$ quando inseridas no transportador $\mathrm{C}_{1}$ encontram a solução do reagente (nitrato de prata $\left.1,0 \times 10^{-2} \mathrm{~mol} \mathrm{~L}^{-1}\right)$ no ponto de confluência $\mathrm{X}$, formando precipitado $(\operatorname{AgBr}(\mathrm{s}))$ no reator helicoidal $\mathrm{B}(200 \mathrm{~cm})$, sendo então monitorado turbidimetricamente em $410 \mathrm{~nm}(\mathrm{E})$.

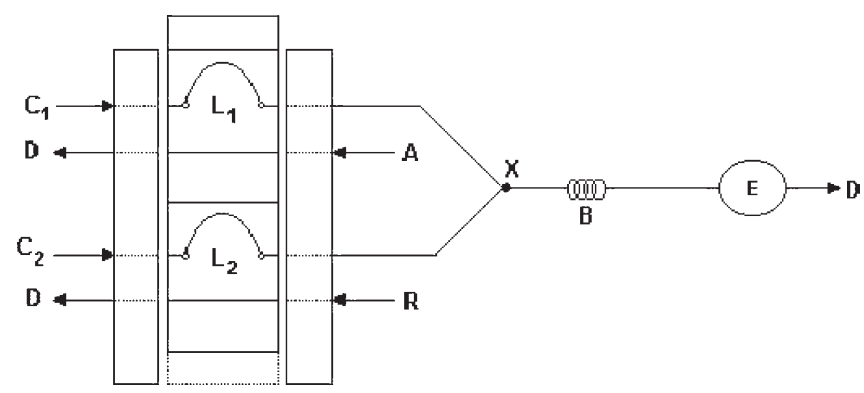

Figura 2. Diagrama esquemático do sistema de análises por injeção em fluxo com zonas coalescentes para determinação turbidimétrica de metilbrometo de homatropina, empregando-se nitrato de prata como reagente de precipitação. $C_{1}=$ transportador da amostra 3,9 $\mathrm{mL} \mathrm{min}^{-1} ; A=$ amostra ou solução de referência; $L_{1}=$ alça da amostra $(375 \mu L) ; C_{2}=$ transportador do reagente 2,0 $\mathrm{mL} \mathrm{min}^{-1} ; R=$ reagente (nitrato de prata $1,0 \times 10^{-2} \mathrm{~mol} \mathrm{~L}^{-1}$ ); $L_{2}=$ alça do reagente $(25 \mu L) ; X=$ ponto de confluência; $B=$ reator helicoidal $(200 \mathrm{~cm}) ; E=$ espectrofotômetro e $D=$ descarte

\section{RESULTADOS E DISCUSSÃO}

\section{Princípio do método turbidimétrico em fluxo}

O metilbrometo de homatropina reage com nitrato de prata, como mostrado na Equação 1, formando $\mathrm{AgBr}_{(\mathrm{s})}$ que foi monitorado em $410 \mathrm{~nm}$.

$\mathrm{C}_{16} \mathrm{H}_{21} \mathrm{NO}_{3} \cdot \mathrm{CH}_{3} \mathrm{Br}_{(\mathrm{aq})}+\mathrm{AgNO}_{3(\mathrm{aq})} \rightleftharpoons\left[\mathrm{C}_{16} \mathrm{H}_{21} \mathrm{NO}_{3} \cdot \mathrm{CH}_{3}\right]\left[\mathrm{NO}_{3}\right]_{(\mathrm{aq})}$
$+\mathrm{AgBr}_{(\mathrm{s})}$

Na otimização do procedimento em fluxo, diversos parâmetros químicos e do sistema em fluxo foram estudados.

\section{Efeito da concentração de $\mathrm{AgNO}_{3}$}

Estudou-se a influência da concentração do reagente nitrato de prata no intervalo de concentrações de $2,5 \times 10^{-3}$ a $5,0 \times 10^{-2} \mathrm{~mol} \mathrm{~L}^{-1}$ sobre o sinal analítico, nas condições experimentais descritas na Figura 2. As melhores sensibilidades e estabilidades da linha base foram obtidas para a concentração de $1,0 \times 10^{-2} \mathrm{~mol} \mathrm{~L}^{-1}$ desse reagente de precipitação, sendo selecionada essa concentração. Para concentrações maiores, houve queda do sinal analítico e perda da estabilidade da linha base, apesar de não ter sido observado entupimento dos canais, fato que pode ocorrer com concentrações mais elevadas do reagente de precipitação.

\section{Efeito do volume da alça de amostragem}

Estudou-se o efeito do volume da alça de amostragem no intervalo de 50 a $500 \mu \mathrm{L}$ para soluções de referência de metilbrometo de homatropina $1,5 \times 10^{-3} ; 2,0 \times 10^{-3}$ e $2,5 \times 10^{-3} \mathrm{~mol} \mathrm{~L}^{-1}$ e concentração de $\mathrm{AgNO}_{3} 1,0 \times 10^{-2} \mathrm{~mol} \mathrm{~L}^{-1}$. Observou-se um aumento do sinal analítico quando o volume da alça de amostra aumentou de 50 a 375 $\mu \mathrm{L}$, permanecendo o sinal analítico constante para volumes de amostra superiores (entre 375 a $500 \mu \mathrm{L}$ ). Sendo assim, selecionouse um volume de amostra de $375 \mu \mathrm{L}$. 


\section{Efeito do volume da alça de reagente}

Estudou-se o efeito do volume da alça de reagente no intervalo de 12,5 a $500 \mu \mathrm{L}$ para soluções de referência de metilbrometo de homatropina $1,5 \times 10^{-3} ; 2,0 \times 10^{-3} \mathrm{e} 2,5 \times 10^{-3} \mathrm{~mol} \mathrm{~L}^{-1}$, concentração de $\mathrm{AgNO}_{3} 1,0 \times 10^{-2} \mathrm{~mol} \mathrm{~L}^{-1}$, vazão do transportador da amostra e reagente de 3,9 e 2,0 $\mathrm{mL} \mathrm{min}^{-1}$, respectivamente, alça de amostragem de $375 \mu \mathrm{L}$ e reator de $50 \mathrm{~cm}$. Como mostra a Figura 3, observou-se maior sinal analítico quando se empregou uma alça de reagente de $25 \mu \mathrm{L}$; para volumes entre 26 e $125 \mu \mathrm{L}$ houve um decréscimo do sinal analítico e, para volumes de alças de reagente maiores o sinal analítico permaneceu praticamente constante. Sendo assim, selecionou-se o volume de $25 \mu \mathrm{L}$ para a alça de reagente, por proporcionar maior sinal analítico e também economia de reagente.

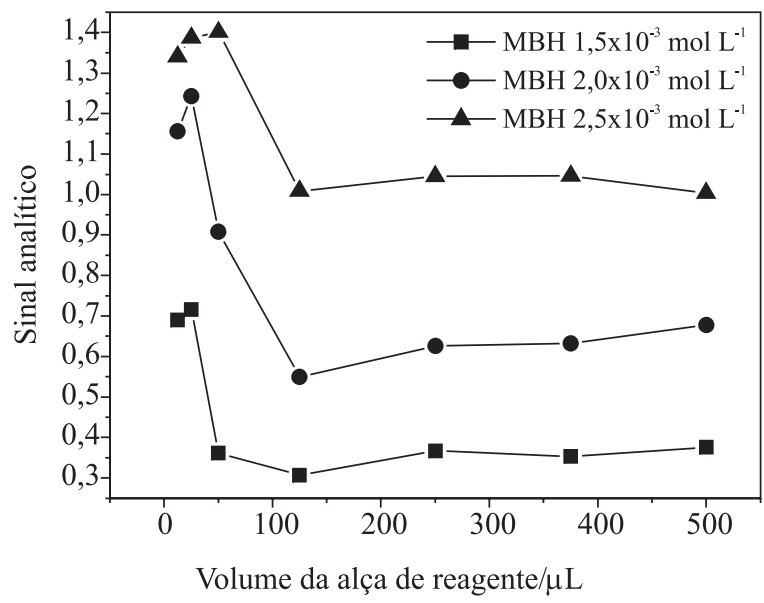

Figura 3. Efeito do volume de alça de reagente de 12,5 a $500 \mu \mathrm{L}$ sobre o sinal analítico para três concentrações de soluções de referência, com vazão de 3,9 $\mathrm{mL} \mathrm{min}^{-1}, \mathrm{AgNO}_{3} 1,0 \times 10^{-2} \mathrm{~mol} \mathrm{~L}^{-1}$, alça de amostra $375 \mu \mathrm{L}$ e reator de $50 \mathrm{~cm}$

\section{Efeito do comprimento do reator}

Estudou-se o efeito do comprimento do reator de 30 a $220 \mathrm{~cm}$ sobre o sinal analítico para soluções de referência de metilbrometo de homatropina $1,5 \times 10^{-3} ; 2,0 \times 10^{-3}$ e $2,5 \times 10^{-3} \mathrm{~mol} \mathrm{~L}^{-1}$ e concentração de $\mathrm{AgNO}_{3} 1,0 \times 10^{-2} \mathrm{~mol} \mathrm{~L}^{-1}$. Observou-se um aumento do sinal analítico até um comprimento de reator de $200 \mathrm{~cm}$, decrescendo em reatores de comprimento maiores, devido provavelmente à dispersão da zona de amostra. Assim, o reator de $200 \mathrm{~cm}$ foi selecionado e empregado nos estudos posteriores.

\section{Efeito da vazão do transportador}

Inicialmente foram realizados alguns estudos objetivando-se obter a melhor sincronicidade do sistema em fluxo. Para isso foi utilizada solução de um composto colorido (hexacianoferrato de potássio (III) $0,01 \% \mathrm{~m} / \mathrm{v}$ ) como solução reagente e água desionizada no lugar da solução de amostra. Um estudo sistemático foi realizado fixando-se a vazão da solução da amostra em $3,9 \mathrm{~mL} \min ^{-1} \mathrm{e}$ variando-se a vazão da solução reagente no intervalo de 1,5 a 4,7 $\mathrm{mL} \min ^{-1}$. Em seguida, a vazão da solução reagente foi fixada em $3,9 \mathrm{~mL} \min ^{-1}$ e a vazão da solução da amostra (água desionizada) foi variada no intervalo de 1,5 a $4,7 \mathrm{~mL} \mathrm{~min}^{-1}$. A melhor sincronicidade foi obtida para vazão de $3,9 \mathrm{~mL} \mathrm{~min}^{-1}$ para solução de amostra e 2,0 $\mathrm{mL} \mathrm{min}^{-1}$ para solução de reagente.

Após este estudo, investigou-se o efeito da vazão do transporta- dor da amostra e reagente sobre o sinal analítico. Este estudo foi implementado fixando-se primeiramente a vazão do transportador do reagente em $3,9 \mathrm{~mL} \mathrm{~min}^{-1} \mathrm{e}$ variou-se a vazão do transportador da amostra de 1,5 a 4,7 $\mathrm{mL} \mathrm{min}^{-1}$. Em seguida, fixou-se a vazão do transportador da amostra em 3,9 mL min ${ }^{-1}$ e variou-se a vazão do transportador do reagente de 1,5 a $4,7 \mathrm{~mL} \mathrm{~min}^{-1}$. Nestes estudos foram utilizadas soluções de referência de metilbrometo de homatropina $1,5 \times 10^{-3} ; 2,0 \times 10^{-3} \mathrm{e} 2,5 \times 10^{-3} \mathrm{~mol} \mathrm{~L}^{-1}$, solução de $\mathrm{AgNO}_{3}$ $1,0 \times 10^{-2} \mathrm{~mol} \mathrm{~L}^{-1}$, alça de amostragem de $375 \mu \mathrm{L}$, alça de reagente de $25 \mu \mathrm{L}$ e reator de $200 \mathrm{~cm}$. Para a amostra foi observado um aumento do sinal analítico ao se aumentar a vazão da solução de amostra de 1,5 a $4,7 \mathrm{~mL} \mathrm{~min}^{-1}$. No entanto, optou-se por trabalhar

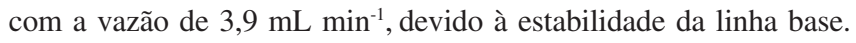
Para o reagente, o sinal analítico diminuiu ao aumentar a vazão da solução de reagente de 2,5 a 4,7 $\mathrm{mL} \mathrm{min}^{-1}$. O melhor compromisso entre freqüência de amostragem, sensibilidade do procedimento em fluxo e estabilidade da linha base foi obtido em vazão da solução de reagente de 2,0 $\mathrm{mL} \mathrm{min}^{-1}$, sendo então selecionada.

Os resultados deste estudo mostraram que empregando-se essas vazões foram obtidos maior sinal analítico, maior estabilidade da linha base e maior freqüência de amostragem.

\section{Estudo do efeito de possíveis interferentes e teste de adição e recuperação}

O estudo de possíveis interferentes na determinação de metilbrometo de homatropina em formulações farmacêuticas foi avaliado para excipientes presentes em amostras comerciais. Neste estudo, foram utilizadas soluções de referência de metilbrometo de homatropina $8,34 \times 10^{-4} \mathrm{~mol} \mathrm{~L}^{-1}$ com cada um dos possíveis interferentes (metilparabeno e propilparabeno), em concentrações de $8,34 \times 10^{-4}$ e $8,34 \times 10^{-3} \mathrm{~mol} \mathrm{~L}^{-1}$. Nenhuma das substâncias investigadas causou qualquer interferência na determinação do metilbrometo de homatropina.

Nos testes de adição e recuperação do analito, três concentrações diferentes de metilbrometo de homatropina, 309,0; 338,0; 367,0 $\mathrm{mg} \mathrm{L}^{-1}$, foram adicionadas a duas amostras de formulações farmacêuticas e os resultados comparados com aqueles obtidos com as amostras não adicionadas do padrão. A recuperação de $\mathrm{MBH}$ variou de 94,9 a $104 \%$, indicando ausência de interferência das matrizes dessas amostras ensaiadas. Os resultados estão apresentados na Tabela 1.

Tabela 1. Estudo de adição e recuperação de metilbrometo de homatropina em formulações farmacêuticas

\begin{tabular}{lccc}
\hline Amostra & \multicolumn{2}{c}{$\begin{array}{c}\text { Metilbrometo de } \\
\text { homatropina }\left(\mathrm{mg} \mathrm{L}^{-1}\right)\end{array}$} & Recuperação (\%) \\
adicionado & encontrado & \\
\hline A & 309,0 & $321,4 \pm 0,9$ & 104 \\
& 338,0 & $331,2 \pm 0,8$ & 97,9 \\
& 367,0 & $348,6 \pm 0,6$ & 94,9 \\
B & 309,0 & $318,3 \pm 0,8$ & 103 \\
& 338,0 & $327,9 \pm 0,7$ & 97,0 \\
& 367,0 & $352,3 \pm 0,9$ & 95,9 \\
\hline
\end{tabular}

\section{Aplicação analítica}

A aplicação analítica do procedimento em fluxo proposto foi realizada empregando-se as melhores condições experimentais encontradas anteriormente, quais sejam: concentração do reagente nitrato de prata $1,0 \times 10^{-2} \mathrm{~mol} \mathrm{~L}^{-1}$, alça de amostragem de $375 \mu \mathrm{L}(75 \mathrm{~cm}) \mathrm{com}$ 
vazão do transportador da amostra de $3,9 \mathrm{~mL} \mathrm{~min}^{-1}$, alça do reagente de $25 \mu \mathrm{L}(5 \mathrm{~cm})$ com vazão do transportador do reagente de $2,0 \mu \mathrm{L}$ $\min ^{-1}$ e reator de $200 \mathrm{~cm}$. A curva analítica foi obtida por injeções sucessivas em triplicata de volumes de soluções de referência de metilbrometo de homatropina, nas concentrações variando de $8,0 \times 10^{-4}$ a $1,7 \times 10^{-3} \mathrm{~mol} \mathrm{~L}^{-1}$. A curva analítica obtida pode ser descrita pela equação: $\mathrm{SA}=-0,978+1,29 \times 10^{3}[\mathrm{MBH}], \mathrm{r}=0,9983$, onde SA é o sinal analítico e $[\mathrm{MBH}]$ é a concentração em mol L-1 de metilbrometo de homatropina. A curva analítica foi linear no intervalo de concentrações supramencionado, com limite de detecção (três vezes o desvio padrão do branco/inclinação da curva analítica) de $9,5 \times 10^{-5} \mathrm{~mol}$ $\mathrm{L}^{-1}$. Os desvios padrão obtidos para solução de metilbrometo de homatropina nas concentrações de $1,2 \times 10^{-3}$ e $1,5 \times 10^{-3} \mathrm{~mol} \mathrm{~L}^{-1}$ foram menores que 2,0\% ( $n=10)$. A freqüência de amostragem foi de $75 \mathrm{~h}^{-1}$, indicando que o sistema pode ser aplicado em laboratórios de controle de qualidade com alta frequiência de amostragem.

Foram analisadas duas amostras, de fabricantes diferentes, cujos resultados empregando o sistema proposto, bem como aqueles obtidos utilizando o método da Farmacopéia Brasileira ${ }^{10}$ são apresentados na Tabela 2. Aplicando-se o teste $t$ pareado ao conjunto de resultados, não foi observada diferença significativa entre os mesmos a um nível de confiança de $95 \%$, indicando a exatidão do procedimento em fluxo desenvolvido. Os sinais transientes obtidos em triplicata para soluções de referência de metilbrometo de homatropina de $8,0 \times 10^{-4}$ a $1,7 \times 10^{-3}$ mol L-1 e soluções de amostras A e B são apresentados na Figura 4.

Tabela 2. Determinação de metilbrometo de homatropina em formulações farmacêuticas pelo método comparativo e pelo método turbidimétrico proposto

\begin{tabular}{lccc}
\hline Amostra & \multicolumn{2}{c}{$\begin{array}{c}\text { Metilbrometo de Homatropina (g/L) } \\
\text { Comparativo }^{10}\end{array}$} & $\begin{array}{c}\text { Erro relativo } \\
\text { Turbidimétrico }\end{array}$ \\
\hline A & $1,98 \pm 0,01$ & $1,94 \pm 0,03$ & $-2,0$ \\
B & $1,97 \pm 0,02$ & $2,01 \pm 0,02$ & 2,0 \\
\hline
\end{tabular}

$\mathrm{n}=3$; *erro relativo entre os métodos turbidimétrico e comparativo.

\section{CONCLUSÕES}

O procedimento de análise por injeção em fluxo proposto nesse trabalho permite a determinação de metilbrometo de homatropina em produtos farmacêuticos utilizando nitrato de prata como reagente precipitante. O método proposto apresenta exatidão, precisão, sensibilidade, pequeno consumo de reagente $\left(0,04 \mathrm{mg}\right.$ de $\mathrm{AgNO}_{3}$ por determinação) e uma freqüência de amostragem de $75 \mathrm{~h}^{-1}$.

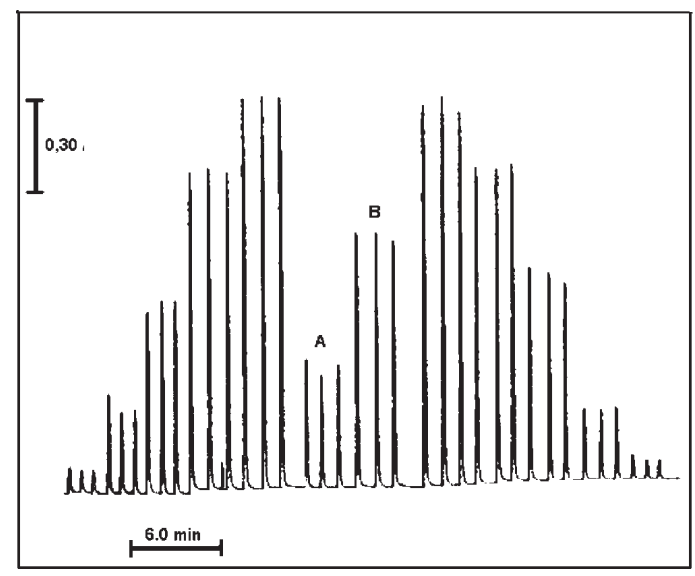

Figura 4. Sinais transientes obtidos em triplicata para soluções de referência de metilbrometo de homatropina, $8,0 \times 10^{-4} ; 1,0 \times 10^{-3} ; 1,2 \times 10^{-3} ; 1,5 \times 10^{-3} \mathrm{e}$ $1,7 \times 10^{-3} \mathrm{~mol} \mathrm{~L}^{-1}$, amostras A e B e soluções de referência em concentrações decrescentes. As condições experimentais foram as mesmas descritas na legenda da Figura 2

\section{AGRADECIMENTOS}

Ao apoio financeiro recebido do CNPq, da CAPES e FAPESP.

\section{REFERÊNCIAS}

1. Goodman, G. A.; Rall, W. T.; Nies, S. A.; The Pharmacological Basis of Therapeutic, $8^{\text {th }}$ ed. , Ed. Mcgraw-Hill: New York, 1992, p. 99-107.

2. Korolkovas, A.; Burckhalter, J. H.; Química Farmacêutica, Guanabara Dois: Rio de Janeiro, 1982, p. 25, 27, 30, 302-30.

3. Patrick, G. L.; An Introduction to Medical Chemistry, Oxford University Press: New York, 1995.

4. Rengstorff, R. H.; Doughty, C. B.; Am. J. Optom. Physiol. 1982, 59, 162.

5. Tune, L. F.; Bylsma, F. W.; Hilt, D. C.; J. Neuropsychiatry Clin. Neurosci. 1992, 4, 195.

6. Majlat, P.; Helboe, P.; Kristensen, A. K.; Int. J. Pharm. 1981, 9, 245.

7. Grabowsk, B. F.; Softly, B. J.; Chang, B. L.; Haney, W. G.; J. Pharm. Sci. 1973, 62, 806.

8. Hanna, S.; Rosen, M.; Rasero, L.; Lachman, L.; J. Pharm. Sci. 1977, 66, 123.

9. Bandelin, F. J.; J. Am. Pharm. Assoc. Sci. 1948, 37, 10.

10. Farmacopéia Brasileira, 2003, método 239.

11. The United States Pharmacopeia- The National Formulary - USP-23, NF18, 1995, p. 744-745.

12. Bergamin, F. H.; Reis, B. F.; Zagatto, E. A. G.; Anal. Chim. Acta 1978, $97,427$. 